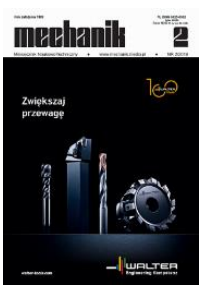

How to cite this article:

Authors: Filip Czubaczyński, Philippe Preumont, Paweł Wittels

Title of article: "Integrated stand for environmental testing of interfaces for gripping and docking used in space robotics

- TesVAC MGSE"

Mechanik, No. 2 (2019)

DOI: https://doi.org/10.17814/mechanik.2019.2.18

\title{
Integrated stand for environmental testing of interfaces for gripping and docking used in space robotics - TesVAC MGSE
}

\author{
FILIP CZUBACZYŃSKI \\ PHILIPPE PREUMONT \\ PAWEE WITTELS*
}

Mgr inż. Filip Czubaczyński, filip.czubaczynski@piap-space.com, - PIAP Space, Polska

Mgr inż. Philippe Preumont, philippe.preumont@piap-space.com - PIAP Space, Polska

Mgr inż. Paweł Wittels, pawel.wittels@piap-space.com - PIAP Space, Polska

The paper describes the concept and mechanical design of applications for environmental testing of interfaces used in space robotics. The state of the art, mechanical description of the proposed solution, functional description, design methodology and tribological problems encountered are discussed. At the end, a summary and scope of further work is presented.

KEYWORDS: ASSIST, LAR gripper, environmental testing, gripping interfaces, docking interfaces

\section{Indroduction}

For the purpose of space research, mechanisms for gripping and docking the target work environments can be simulated in laboratories on Earth.

There are many types of simulators that use computer and physical models. Among the simulators using physical models, robotic stands for dynamic and thermal-vacuum tests can be mentioned.

Mobile robots are most often used in dynamic tests in one plane. An example of such a system is the space robot simulator at NTUA Control Systems Laboratory (fig. 1) [1, 2].

It consists of active and passive platforms. The platforms move on a granite table ensuring a flatness of 5 $\mu \mathrm{m}$ and low surface roughness. The robots move on air cushions, which allows the simulation of natural buoyancy occurring in orbit. The limitation of this test stand is the ability to move only in one plane.

Industrial robots allow testing in 3D space. Examples of such applications include the platform-art(C) developed by GMV (fig. 2) [3]. It consists of two industrial robots. One is fixed and the other is on the guide. An example of a temperature and pressure testing application is the Linear Slide Assembly.

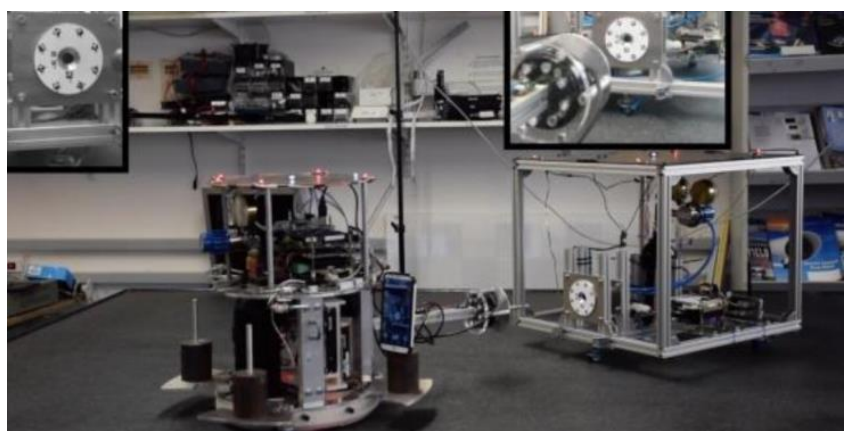

Fig. 1. NTUA Space Robot Simulator (photo by NTUA) [2] 


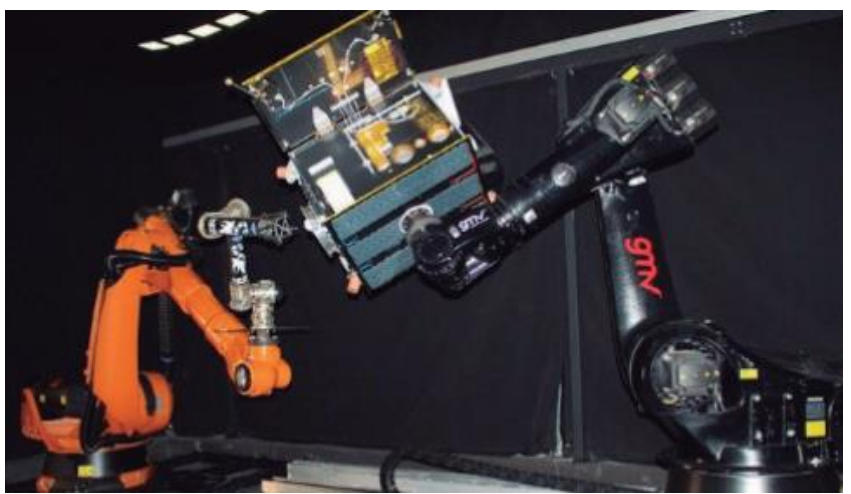

Fig. 2. GMV platform-art ${ }^{\circledR}$ (photo by GMV) [3]

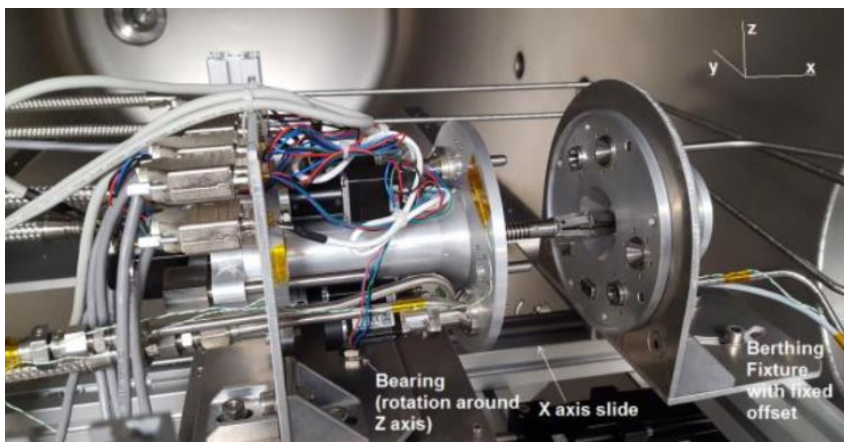

Fig. 3. Application for environmental tests (photo by Moog Space \& Defense Group) [2]

During the tests, the device is placed in a thermal-vacuum chamber (fig. 3) [2]. It is possible to set angular $(Z$ axis) and linear ( $Y$ axis) deviations, and then simulate the proximity of two parts of the interface under test. There are mechanical, electrical and hydraulic interfaces. These applications do not allow dynamic tests with angular deviation in three axes under given thermal and vacuum conditions.

\section{Research object}

\section{- System requirements}

The goal of the research is to develop an integrated stand enabling testing of thermal-vacuum interfaces for gripping and docking used in space robotics. When connecting the two parts of the interface under test, active measurements of forces and moments in all axes will be carried out.

This system will be used in tests of interfaces developed by the European Space Agency (ESA), designed for e.g. capturing satellites (mission e.Deorbit) or servicing satellites in orbit (ASSIST project).

The main requirements that the device should meet:

- operating temperature range: -40 to $+80^{\circ} \mathrm{C}$,

- automatic operation (min. $8 \mathrm{~h}$ ) in vacuum, up to 10-6 mbar,

- ability to record test results,

- possibility of deviating part B of the tested interface, mounted on the gimbal: angular $\pm 15^{\circ}$ in three axes and linear $\pm 24 \mathrm{~mm}$ in the $Y$ axis,

- possibility of generating up to $80 \mathrm{~N}$ force and $20 \mathrm{Nm}$ torque when connecting interfaces,

- active measurement of generated forces in two ranges: $0 \div 200 \mathrm{~N}$ and $0 \div 25 \mathrm{~N}$, in three axes and measuring accuracy of $10 \%$,

- compatibility with available test infrastructure,

- modular design allowing testing of many types of interfaces,

- mass of individual modules allowing them to be carried by one person.

\section{- Definitions}

For simplicity, the interfaces tested are divided into Part A and Part B. They can be static or motorized. Part $\mathrm{A}$ is mounted on the fixed rack module. Part B is mounted on the motorized gimbal module. 


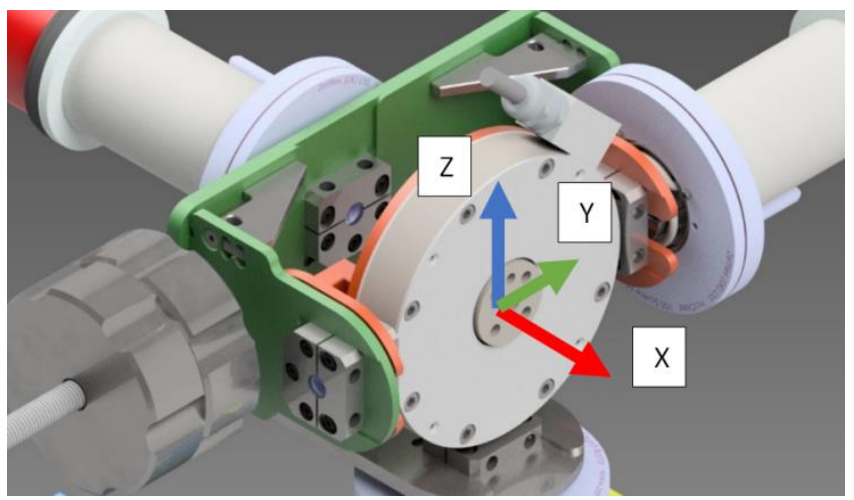

Fig. 4. Joint mounting interface for part B with the reference system marked on it (photo by PIAP Space)

The following reference system was adopted (fig. 4):

- the $X Z$ plane is perpendicular to the test table,

- the $X Z$ plane contains the $X$ axis of the propeller in the $X$ table

- the $X Y$ plane is parallel to the surface of the test table,

- the attachment point is in the center of the force and torque sensor.

- Mechanical design of the device

The device is divided into (fig. 5):

- rack module (A) designed to store part A (D) of the interface being tested,

- gimbal module (B) designed to manipulate part B (E) of the interface being tested,

- mechanical relative positioning interface (C),

- control unit and wiring harness (not shown).

The rack module consists of:

- frames with interfaces for attaching the vacuum chamber to the table, positioning the gimbal module and grounding points,

- removable mounting plate with mounting interface for part A.

Additional plates can be attached to it to extend its functionality.

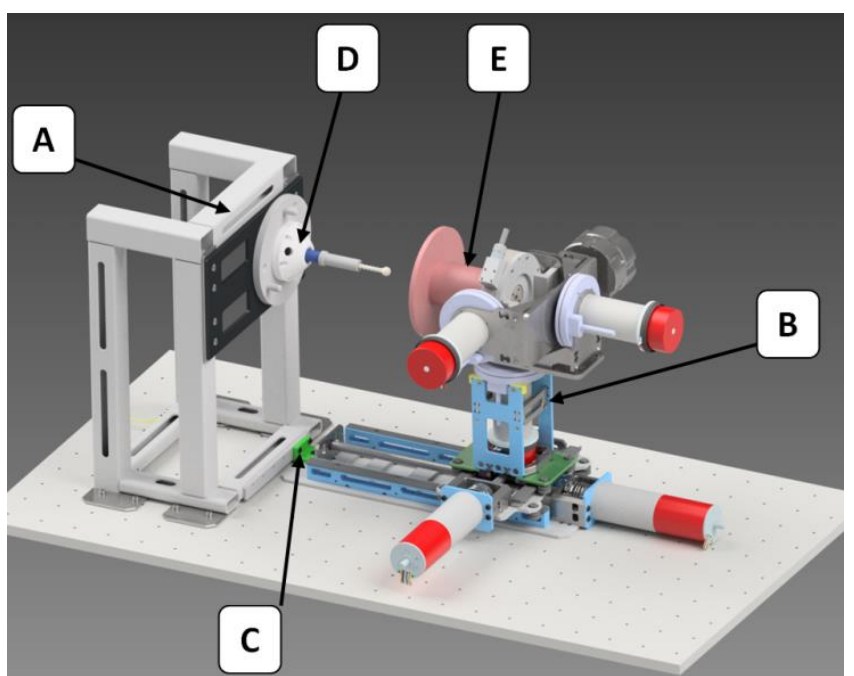

Fig. 5. General view of the device (photo by PIAP Space) 


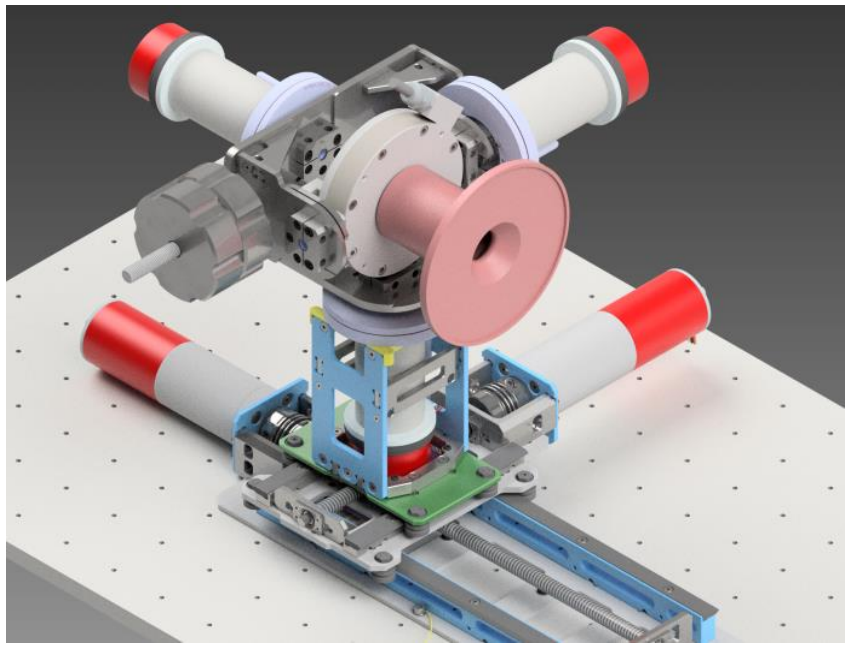

Fig. 6. Gimbal module (photo by PIAP Space)

The gimbal module consists of (fig. 6):

- the $X Y$ table, responsible for the linear misalignment of the B part ( $Y$ axis); it enables linear movement of the B part in the $X$ axis $(200 \mathrm{~mm}$ stroke + additional $10 \mathrm{~mm}$ ) and in the $Y$ axis (48 $\mathrm{mm}$ stroke + additional $10 \mathrm{~mm}$ ) and has an interface for mounting to the table of the thermal-vacuum chamber,

- the three-axis gimbal, which is responsible for handling and setting the initial angular misalignment of part B in all controlled axes up to $\pm 15^{\circ}$, has an interface for attachment to the previously mentioned $X Y$ table.

\section{Design methodology and scope of research}

\section{- Design methodology}

The device specification was determined on the basis of the interface descriptions developed by ESA. The research infrastructure was reviewed. The mechanical design including 3D CAD model of the proposed position was presented. Based on this, the initial mechanical design of the device was developed.

At this stage, several proposed solutions have not been specified. Compliance with the specifications was confirmed in the preliminary design review (PDR). The initial model has been refined. The final mechanical design was created. After confirming its compliance with the specification, the implementation documentation was prepared at the critical design review (CDR). Its compliance has been confirmed at the production readiness review (MRR).

Production of parts and purchase of commercial components were commissioned. After collecting all the elements, the assembly of the station will be carried out. The prototype will be subject to test readiness review (TRR). Test results will be accepted for review before the college (TRB). The device will be finally picked up at the review confirming readiness to hand over to the customer (DRB).

\section{- Tribological problems}

The challenge was the selection of materials and coatings for standard parts, commercial components, greases, adhesives, insulation and solder for use in predefined conditions. The main selection parameter was the lowest possible degassing factor value of the materials used. When selecting coatings, care was taken to avoid adhesive seizing and cold welding between the cooperating elements.

To reduce the cost of manufacturing the device, industrial BLDC motors were used, subjected to some modifications. They consisted of:

- replacement of electric wires,

- replacement of grease in motor bearings and the planetary gear attached to it.

An interesting component is the flexure pivot (fig. 7). It can replace conventional bearings in pivot joints with limited movement range. Friction-free operation is a huge advantage in the absence of air during testing. 


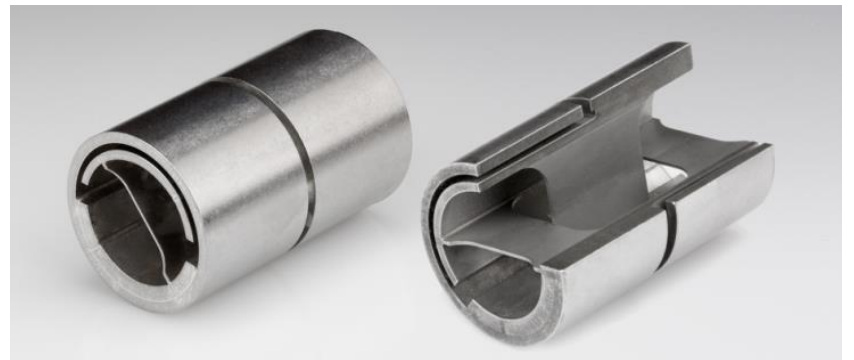

Fig. 7. Flexible pivot; on the left - whole, on the right - cut (photo by NASA)

\section{Summary and directions of further work}

The device can be used to perform interface tests:

- LAR (Launch Adapter Ring) and gripper for LAR or similar interface (fig. 8),

- docking probe and its socket (ASSIST),

- androgynous interfaces to the modular spacecraft design (SIROM).

- Further work will include:

- prototype design, mechanisms verification,

- internal tests in the atmosphere, followed by a thermal-vacuum chamber,

- tests of various interfaces in their target work environment.

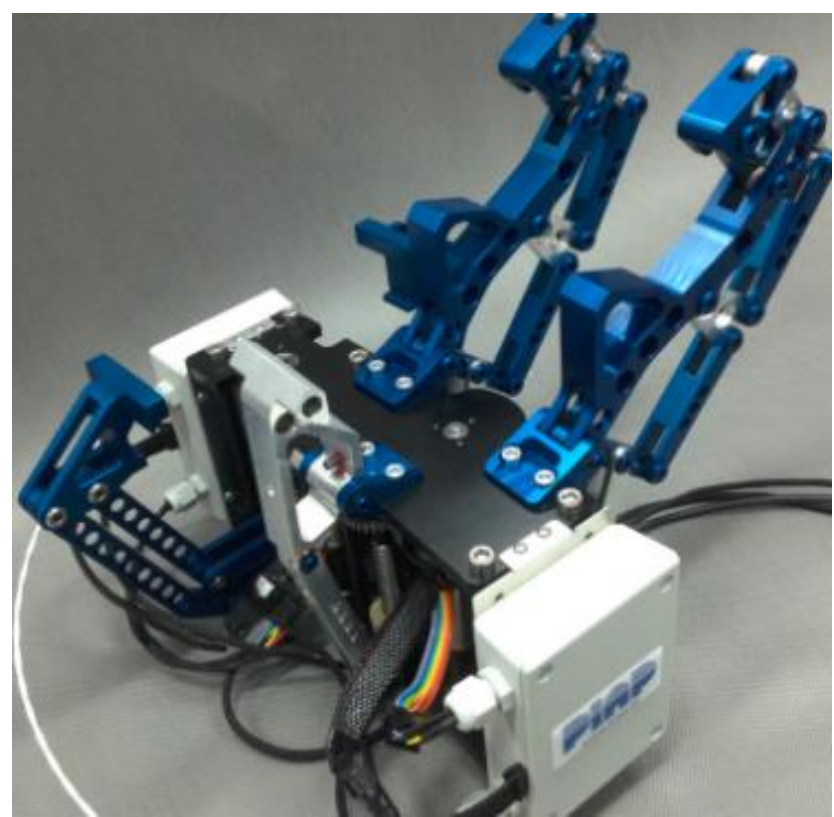

Fig. 8. LAR gripper (photo by PIAP)

\section{Acknowledgments}

The device was designed as part of the TesVAC project, which is implemented as part of the Polish Industry Incentive Scheme program.

\section{REFERENCES}

[1] Papadopoulos E., Paraskevas I., Flessa T., Nanos K., Rekleitis G., Kontolatis I. „The NTUA space robot simulator: design \& results". 10th ESA Workshop on Advanced Space Technologies for Robotics and Automation (ASTRA'08), ESA, ESTEC, Noordwijk, Holandia, 2008.

[2] Tomassini A., Solway N., Rekleitis G., Papadopoulos E., Krenn R., Rohrbeck M., Vidal Ch., Delage R., Hobbs L., Aziz S., Visentin G. "Testing and cross-validation of on-orbit servicing system for geo spacecraft refuelling". 19th ESA Workshop on Advanced Space Technologies for Robotics and Automation (ASTRA'17), ESA, ESTEC, Noordwijk, Holandia, 2017.

[3] https://www.gmv.com/en/Products/platform/ (access: 07.12.2018).

[4] ECSS-M-ST-10C Rev.1 - Project planning and implementation (06.03.2009). 